\title{
Lung cancer inhibitory activity of dietary berries and berry polyphenolics
}

\author{
Farrukh Aqil $^{\mathrm{a}, \mathrm{b}}$, Jeyaprakash Jeyabalan ${ }^{\mathrm{b}}$, Hina Kausar ${ }^{\mathrm{b}}$, Radha Munagala ${ }^{\mathrm{a}, \mathrm{b}}$, Inder Pal Singh ${ }^{\mathrm{d}}$ \\ and Ramesh Gupta ${ }^{\mathrm{b}, \mathrm{c}, *}$

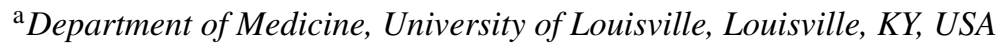 \\ ${ }^{\mathrm{b}}$ James Graham Brown Cancer Center, University of Louisville, Louisville, KY, USA \\ ${ }^{\mathrm{c}}$ Department of Pharmacology and Toxicology, University of Louisville, Louisville, KY, USA \\ ${ }^{\mathrm{d}}$ National Institute of Pharmaceutical Education and Research, S.A.S Nagar, India
}

Received 24 November 2015; accepted 31 January 2016

\begin{abstract}
.
BACKGROUND: Blueberry (BB) and black raspberry (BRB) have been shown to be chemopreventive against estrogenmediated breast cancer in pre-clinical studies. However, therapeutic efficacy of these berries against lung cancer is not known.

METHODS: In this study we investigated i) relative efficacy of individual anthocyanidins vs. respective anthocyanins, ii) relative antiproliferative activity of mixture of anthocyanidins compared to individual anthocyanidins, iii) antitumor activity of dietary BB, iv) Tumor inhibitory activity of diet supplemented with BB, alone and in combination with BRB, against lung tumor xenograft using nude mice, and finally, v) the efficacy of select polyphenolics present in BB and BRB against lung tumor xenograft.

RESULTS: Our findings indicated that individual anthocyanidins (aglycones) were significantly more potent (2-3 fold lower $\mathrm{IC}_{50}$ ) in inhibiting the non-small-cell lung cancer (NSCLC) cell growth vs. respective anthocyanins (glycones). Further, anthocyanidins mixture at equimolar concentrations exhibited synergistic antiproliferative activity vs. individual anthocyanidins. When tested against NSCLC (A549 and H1299) cells in nude mice, dietary BB (7.5\%, w/w) showed $>40 \%$ reduction in tumor volume against H1299 xenografts. The maximal growth inhibition occurred with 5\% BB dose, with no additional protection occurring at a higher dose $(7.5 \%)$. However, somewhat lower protection was found when the BB diet initiated prior to tumor cell inoculation. The mixture of BB $(5 \%, \mathrm{w} / \mathrm{w})$ and BRB $(2.5 \%)$ resulted in higher inhibition of tumor growth vs. BB alone ( $71 \%$ vs $42 \%$ ). Likewise, a combination of delphinidin (bioactive of BB) and punicalagins (a bioactive of BRB, which gets converted to ellagic acid in vivo) showed higher tumor growth inhibition compared to delphinidin.

CONCLUSIONS: The therapeutic effects of the berries and berry polyphenolics observed against lung cancer in this study are highly encouraging. Further investigation into the mechanism of action of the combinations of the berry bioactives will be valuable for clinical use of this potent natural product against lung cancer.
\end{abstract}

Keywords: Berries, anthocyanins, anthocyanidins, lung cancer, tumor xenograft

\footnotetext{
*Corresponding author: Ramesh C. Gupta, Delia Baxter II, Room 304E, University of Louisville, Louisville, KY, USA. Tel.: +1 502 852 3682; E-mail: rcgupta@louisville.edu.
}

1878-5093/16/\$35.00 @ 2016 - IOS Press and the authors. All rights reserved

This article is published online with Open Access and distributed under the terms of the Creative Commons Attribution Non-Commercial License. 


\section{Introduction}

Lung cancer is the leading cause of cancer-related deaths in US and worldwide with an estimated 1.3 million total deaths annually [1]. Unlike significant progress made in the prognosis of certain cancers (e.g., breast, prostate), the prognosis for lung cancer remains grim. The 5-year survival for lung cancer still hovers around $15 \%$. Non-small-cell lung cancer (NSCLC) predominates (85\%) of all lung cancers. Lung cancer diagnosed with stage I represents only a small fraction (about $15 \%$ ). Following resection, 30\% - 50\% of these patients develop recurrence within 5 years [2]. The situation is dismal for late stage lung cancer patients. Unfortunately, there are no standard practices per se for the prevention of lung cancer recurrence and metastasis; research in this arena is also limited. There is a great need for some unconventional, user-friendly approaches to improve the treatment of lung cancer and prevent or delay the recurrent lung disease.

Dark-colored fruits and vegetables have generally been accepted as cancer preventive. Berries have received great attention lately based on their potential to prevent chemically-induced colon [3] and esophageal [4] cancers in animal models. The initial evidence that berry anthocyanins are chemopreventive in an animal tumor-model was provided by Wang et al. [5, 6]. This study also demonstrated that the anthocyanin fraction of black raspberries (BRB) was as effective in preventing chemically-induced esophageal tumors in rats as whole BRB powder [6]. Our own studies have shown significant inhibition of breast cancer in the rat model by both blueberry (BB) and $\mathrm{BRB}$, indicating that berries are chemopreventive beyond the GI tract [7-9]. Our studies with BB diet have also shown that berry anthocyanidins are bioavailable beyond the GI as we could detect all anthocyanidins in lung tissues [10]. The berry bioactives including anthocyanins also have significant therapeutic activity against breast cancer in vivo [11].

The chemopreventive and therapeutic activity of BB has been attributed to the presence of polyphenolics, specifically anthocyanins (Fig. 1). On the other hand, Wang et al. [6] have also reported chemopreventive activity of the fiber fraction of blueberries against the esophageal tumorigenesis. Evidence suggest anthocyanins possess several beneficial attributes such as antioxidant and anti-inflammatory effects, reduce risk of stroke and coronary diseases, and improve cognitive behavior [12]. Further, aglycones of anthocyanins isolated from Indian blackberry (Jamun) demonstrated higher antiproliferative activity compared with anthocyanins against lung cancer A549 cells in vitro [13]. The clear evidence of the role of anthocyanidins as the presumptive BB bioactives came from our recent study in which native mixture of anthocyanidins isolated from bilberry showed significant inhibition of human lung cancer cells both in vitro and in vivo [14].

In this study, we tested relative antiproliferative activity of individual as well as mixture of anthocyanidins and compared with respective anthocyanins and their mixture in cell culture. BB and BRB contain different profile of phytocompounds. BB contains glycones of five distinct anthocyanidins, whereas BRB contains glycones of almost exclusively cyanidin together with a high amount of EA/ellagitannins. We therefore tested diet supplemented with whole BB powder, alone and in combination with BRB powder for antitumor activity against lung cancer tumor xenograft using nude mice.

\section{Materials and methods}

\subsection{Chemicals}

High-performance liquid chromatography (HPLC)-grade water, acetonitrile, methanol and other HPLC solvents, and hydrochloric acid $(\mathrm{HCl})$ were obtained from Sigma-Chemical Co. (St. Louis, MO). Authentic anthocyanins standards were obtained from Chromadex (Irvine, CA). All other chemicals used in the study were of analytical grade. Cyanidin and cyanidin-3-glucoside (99\%) were provided by Medox, Norway as kind gift. 


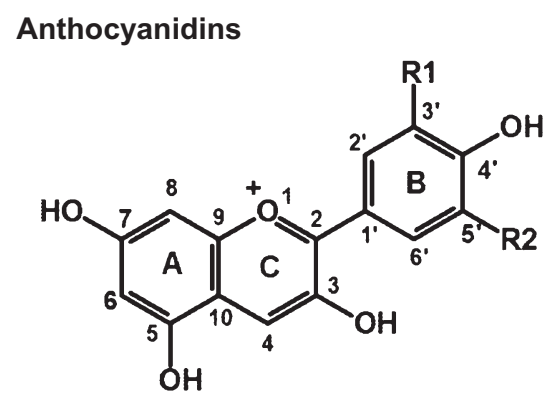

\begin{tabular}{|l|l|l|}
\hline & $\mathbf{R}_{1}$ & $\mathbf{R}_{2}$ \\
\hline Delphinidin & $-\mathrm{OH}$ & $-\mathrm{OH}$ \\
\hline Cyanidin & $-\mathrm{OH}$ & $-\mathrm{H}$ \\
\hline Peonidin & $-\mathrm{OCH}_{3}$ & $-\mathrm{H}$ \\
\hline Petunidin & $-\mathrm{OH}$ & $-\mathrm{OCH}_{3}$ \\
\hline Malvidin & $-\mathrm{OCH}_{3}$ & $-\mathrm{OCH}_{3}$ \\
\hline
\end{tabular}

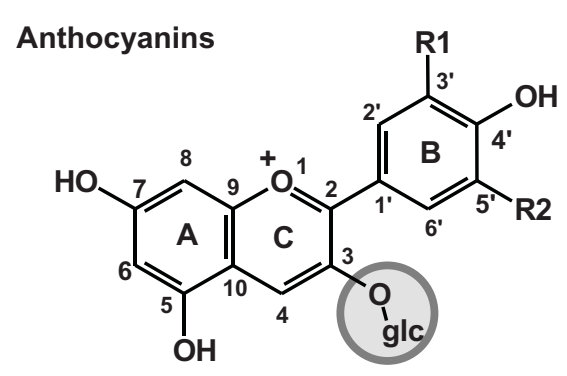

Anthocyanin monoglucoside

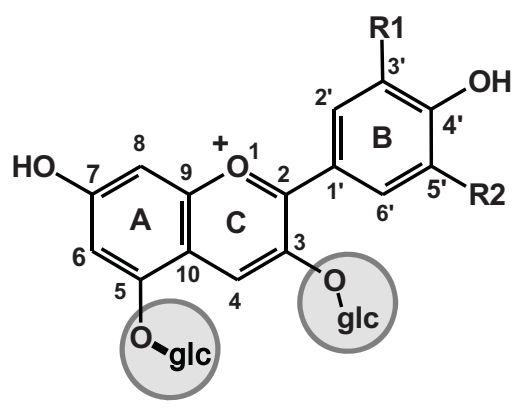

Anthocyanin diglucoside

Fig. 1. Structures of various anthocyanidins and anthocyanins.

\subsection{Berry powder}

Freeze-dried highbush BB powder (50:50 blend of tifblue and rubel) was received from the U.S. Highbush Blueberry Council (Folsom, CA). BRB was procured as freeze-dried powder (Van Drunen farms, Momence, IL) through Dr. Gary Stoner. The native mixture of anthocyanidins in BB and bilberry are essentially the same and contains five major anthocyanidins, namely delphinidin (Dp), cyanidin (Cy), malvidin (Mv), peonidin (Pe) and petunidin $(\mathrm{Pt})$ in the relative ratio of $33: 28: 16: 16$ and 7 as described [14]. We therefore used bilberry to isolate anthocyanidins as this material is available in highly enriched extract form.

\subsection{Extraction and isolation of purified anthocyanidins from bilberry}

The native mixture of anthocyanidins ( $\sim 95 \%$ pure) was isolated in our laboratory from $36 \%$ anthocyaninenriched bilberry extract (Indena, Seattle, WA). Extraction, enrichment and hydrolysis of the bilberry were carried out using essentially the same method as described previously $[14,15]$. Briefly, the enriched bilberry extract powder was extracted with $75 \%$ aqueous ethanol containing $0.1 \% \mathrm{HCl}$ and enriched by loading the concentrated extracts on a XAD-761/diaion HP-20 $(1: 1)$ column. The polyphenols, including anthocyanins were eluted with methanol. Pooled eluates were concentrated and hydrolyzed with $2 \mathrm{~N} \mathrm{HCl}(\sim 5 \mathrm{vol})$. Hydrolysates were purified using C18 Sep-Pack cartridges (Waters, Milford, MA). Anthocyanidins and other polyphenols were eluted with acidified $(0.01 \% \mathrm{HCl})$ methanol. The enriched extracts were dried under reduced pressure using a Savant Speed-Vac (Thermo Scientific, USA) and stored at $-20^{\circ} \mathrm{C}$ until use. 


\subsection{Diet}

AIN-93M diet supplemented with BB powder at 2.5\% and 5\% (w/w) was prepared in pellet form by HarlanTeklad (Madison, WI) and stored at $4{ }^{\circ} \mathrm{C}$ in dark in vacuum-sealed bags until use. For the preparations of the $\mathrm{BB}$ and BRB diet, AIN 93M diet was powdered and mixed with BB and BRB powder at $5 \%$ and $2.5 \%(\mathrm{w} / \mathrm{w}$ ), respectively, and stored at $4{ }^{\circ} \mathrm{C}$ until use. The diets were isocaloric and the recipe used was the same as described by us elsewhere $[8,16,17]$.

\subsection{HPLC analysis}

HPLC analysis was carried out according to Li et al. [18] with minor modifications on a Shimadzu HPLC system (Kyoto, Japan) equipped with two LC-10ADvp pumps, an autosampler, fluorescent and diode array detectors and operated by Class VP (ver 7.4 SP3). BB and BRB extracts $(20 \mu \mathrm{L}$ injection volume; $1 \mathrm{mg} / \mathrm{ml}$ ) were analyzed on a ShimPack reverse phase column (Shimadzu; $250 \times 4.6 \mathrm{~mm}, 2.2 \mu \mathrm{m}$ ). Two separate experiments were carried out using different gradients of $3.5 \%$ aqueous phosphoric acid (solvent A) and acetonitrile (solvent B). The first experiment was used to separate all anthocyanidins, and the second was used for the separation of anthocyanins, anthocyanidins, and other polyphenolics including EA and ellagitannins.

The first linear gradient contained solvent A initially $95 \%$ for $10 \mathrm{~min} ; 90 \%$ at $20 \mathrm{~min} ; 87 \%$ at $30 \mathrm{~min} ; 82 \%$ at $35 \mathrm{~min} ; 75 \%$ at $40 \mathrm{~min} ; 40 \%$ for $41-61 \mathrm{~min}$; and finally $95 \%$ in acetonitrile at a flow rate of $0.75 \mathrm{ml} / \mathrm{min}$. The total run time was $61 \mathrm{~min}$ with $10 \mathrm{~min}$ intervals between the injections. Anthocyanidins were monitored at $520 \mathrm{~nm}$. The second gradient contained solvent A initially $90 \%$ for $0-5 \mathrm{~min} ; 85 \%$ at $10 \mathrm{~min} ; 80 \%$ at $15 \mathrm{~min} ; 70 \%$ at $24 \mathrm{~min} ; 62 \%$ at $35 \mathrm{~min}$; $94 \%$ for $40-43 \mathrm{~min}$; and finally $90 \%$ at $45 \mathrm{~min}$. EA/ellagitannins and polyphenolics were monitored at 366 and $280 \mathrm{~nm}$, respectively. Anthocyanidins and EA peaks were confirmed by co-chromatography with the reference materials.

\subsection{Cell proliferation assay and measurements of cell viability}

Inhibition of cell proliferation by berry anthocyanins and anthocyanidins was measured in the human NSCLC cell line A549. Cells were procured from the American Type Culture Collection (ATCC) and maintained in DMEM supplemented with $10 \%$ fetal bovine serum, $100 \mathrm{units} / \mathrm{ml}$ penicillin and $100 \mu \mathrm{g} / \mathrm{ml}$ streptomycin. Cells were plated in 96-well culture plates at $3 \times 10^{3}$ cells/well. After $24 \mathrm{~h}$, cells were treated with individual or a mixture of anthocyanins and anthocyanidins for $72 \mathrm{~h}$, after which cell density was measured using MTT assay [19].

\subsection{Effect on tumor xenograft}

Three studies were conducted in athymic nude mice (Athymic Nude-Foxn $I^{\text {nu }}$ ) to analyze the efficacy of BB and BRB. All the studies followed the same protocol except that diets were supplemented with the berry powder at different doses. Briefly, female nude mice (4-5-wk old) were purchased from Harlan, acclimated for a week on a chow diet followed by control AIN-93M diet for another week. The animals were then inoculated with human lung cancer H1299 $\left(1.5 \times 10^{6}\right)$ or A549 $\left(3 \times 10^{6}\right)$ cells in matrigel to establish subcutaneous xenografts. When the tumor xenografts grew to about $80 \mathrm{~mm}^{3}$, the animals were randomized into different groups and were continued either on the control diet or switched to experimental diets. In the first study, athymic nude mice bearing human lung cancer H1299 cells xenograft were treated with a diet supplemented with $7.5 \%$ BB. In the second study, different doses of BB powder were tested against human A549 lung cancer xenograft. Diets supplemented with $2.5 \%$ and $5 \%$ of $\mathrm{BB}$ powder were provided either in pre-inoculation stage - four days before inoculating with the tumor cells, or in post-inoculation stage after tumors grew to $\sim 80 \mathrm{~mm}^{3}$. In our third study, we tested the combination of BB (5\%,w/w) and BRB $(2.5 \%, \mathrm{w} / \mathrm{w})$ against A549 lung tumor xenograft. Diet intake and body weight were monitored weekly along with measurement of the tumor size by digital caliper. The animals were euthanized when the tumors grew to $800-1,200 \mathrm{~mm}^{3}$ by $\mathrm{CO}_{2}$ asphyxiation. 

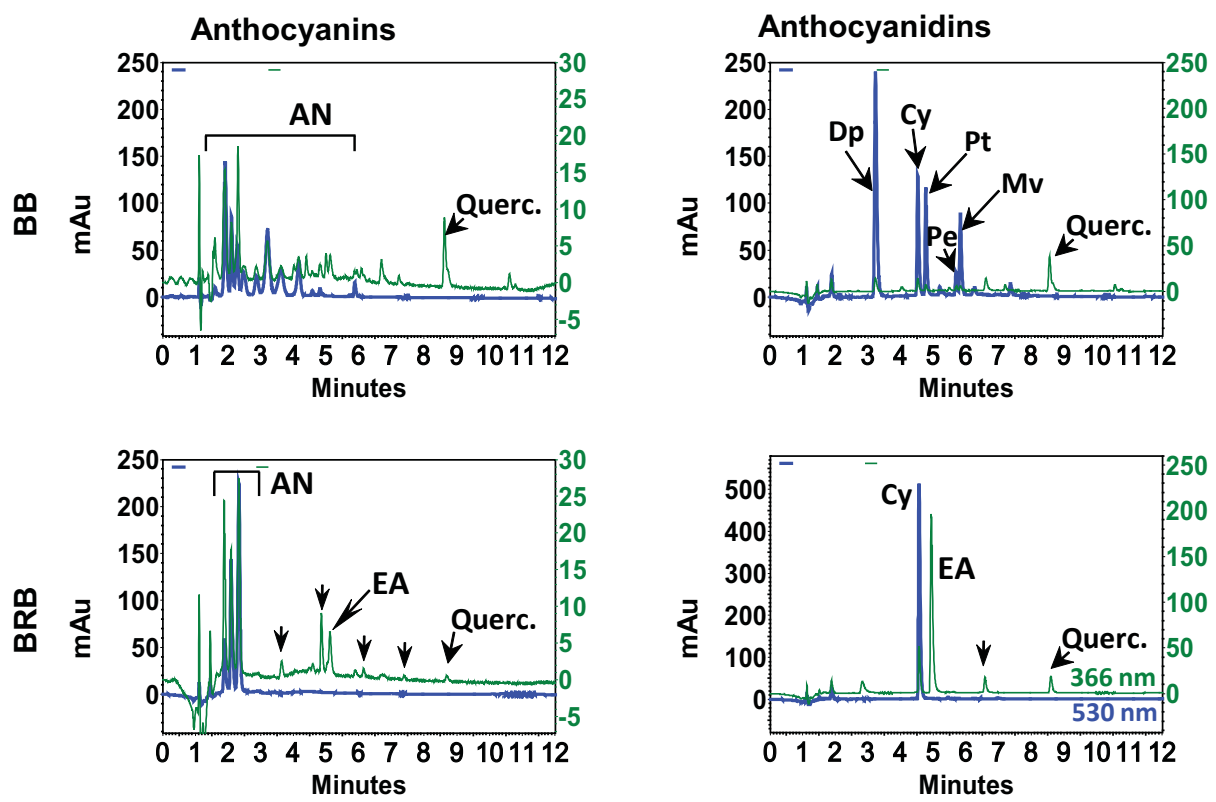

Retention time

Fig. 2. HPLC profiles of blueberry (BB) and black raspberry (BRB). Enriched hydrolyzed and non-hydrolyzed extracts ( $20 \mu \mathrm{l} ; 1 \mathrm{mg} / \mathrm{ml})$ were injected on a reverse phase $\mathrm{C} 18(250 \times 4.6 \mathrm{~mm} \mathrm{ID} \times 5 \mu \mathrm{m})$ column in a gradient of acetonitrile and $3.5 \%$ aqueous phosphoric acid and analyzed using a photo diodarray detector in the range of $210-550 \mathrm{~nm}$. Chromatograms in the first column represent anthocyanins and other phenolics including free ellagic acid (EA) and quercetin monitored at 530 and $366 \mathrm{~nm}$, respectively. Chromatograms in the second column show anthocyanidins and other phenolics including free EA and quercetin. mAu, milliabsorbance units. Partially adopted from Journal of Agricultural and Food Chemistry, 2014, 62 (18), Aqil et al., Detection of anthocyanins/anthocyanidins in animal tissues, 3912-3918 with per-mission from American Chemical Society, Copyright (C) 2014.

\subsection{Statistical analysis}

All statistical analyses were performed with GraphPad Prism statistical software (version 4.03; La Jolla, CA). For cell proliferation assay, $\mathrm{IC}_{50}$ values were calculated using Microsoft Excel, XL Fit version 5 software. Data represented in the xenograft studies is the average \pm standard error of 8-10 replicates. Differences between the means of the treatments were calculated for tumor volume and $p$-values were determined by Student's $t$-test. Differences were considered a priori to be statistically significant if the $P$ value was less than 0.05 .

\section{Results and discussion}

Berries are gaining increased attention lately and it is now generally accepted that berry bioactives (anthocyanins) are the most important group of water-soluble pigments in plants. There is a vast literature on their occurrence and health benefits including a substantial number of reviews. We have demonstrated the chemical profile and biological efficacy of BB and BRB against hormonal mammary tumorigenesis in ACI rats [20]. HPLC profiles of anthocyanins and anthocyanidins indicated BB and BRB contained several anthocyanins (Fig. 2). Upon acid hydrolysis, BB showed the presence of five distinct anthocyanidins ( $\mathrm{Dp}, \mathrm{Cy}, \mathrm{Mv}, \mathrm{Pe}$ and $\mathrm{Pt}$ ) while BRB contained almost exclusively $\mathrm{Cy}$ in addition to EA, a well-known chemopreventive agent (Fig. 2). 
A

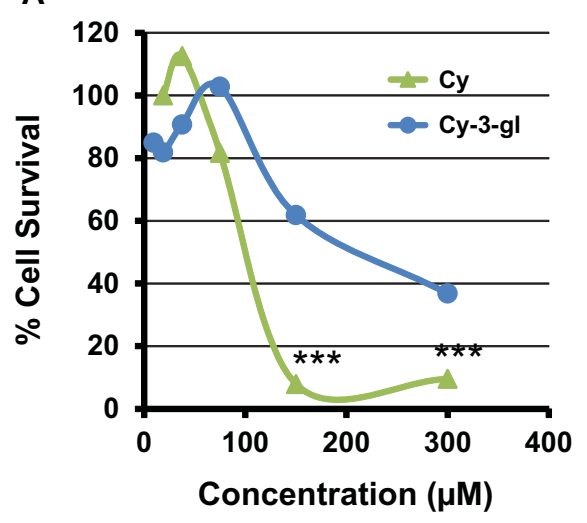

B

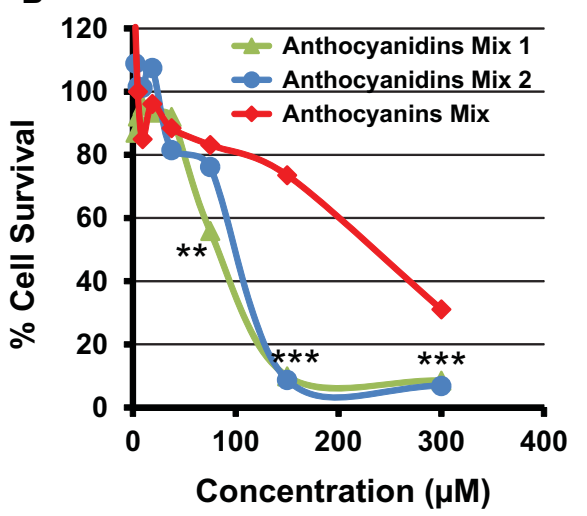

Fig. 3. Antiproliferative activity of cyanidin and cyanidin-3-glucoside by MTT assay against human lung A549 cells (A). Panel B shows the effect of native mixture of BB anthocyanidins ( $\mathrm{Cy}, \mathrm{Mv}, \mathrm{Pe}, \mathrm{Pt}$ and $\mathrm{Dp}$ ) (Anthocyanidins Mix 1), and their equimolar mixture on cell viability of human lung cancer cells (Anthocyanidins Mix 2). The effect of anthocyanidins was compared to the equimolar anthocyanins mixture (Anthocyanins Mix). Data are expressed as percentage of untreated cells. Standard deviation of (5-7\%) is not shown for clarity.

To understand differences between the activity of anthocyanins and anthocyanidins, antiproliferative activity of cyanidin-3-glucoside was tested against lung cancer A549 cells and compared with its aglycone counterpart, Cy. Cy showed almost 2 -fold reduction in the dose to inhibit $50 \%$ of the cancer cells (Fig. 3A) compared to cyanidin3 -glucoside. Similarly, an equimolar mixture of five anthocyanidins showed higher potency $\left(\mathrm{IC}_{50}=102 \mu \mathrm{M}\right)$ compared with the mixture of their respective anthocyanins $\left(\mathrm{IC}_{50}=265 \mu \mathrm{M}\right)$. Also, native mixture of BB anthocyanidins demonstrated similar potency as $1: 1$ equimolar mixture of anthocyanidins (Fig. 3B). The higher activity of the anthocyanidins compared to anthocyanins presumably occurred due to their higher cellular uptake. These findings are in agreement with our previous findings where we demonstrated similar higher activity from enriched extract prepared at anthocyanidins level (hydrolyzed) compared to anthocyanins level (non-hydrolyzed) of Indian blackberry, jamun against lung cancer cells in vitro [15]. Furthermore, our recent studies showed that a combination of $\mathrm{Dp}, \mathrm{Cy}, \mathrm{Pe}, \mathrm{Pt}$ and $\mathrm{Mv}$ produced synergistic antiproliferative effect compared to individual entities [14]. This enhanced activity resulted presumably due to the attack of these anthocyanidins at distinct and overlapping targets [14].

The efficacy of diet supplemented with BB powder against the growth of human lung cancer cells was determined using three different animal studies. In our first study, athymic nude mice bearing highly aggressive human lung cancer H1299 cells xenograft were treated with a diet supplemented with $7.5 \%$ BB. No significant differences were observed in the diet intake and the body weight gains until the end of the study indicating that the BB diet was well tolerated. The BB diet showed a significant inhibition of the lung tumor growth compared to the control diet $\left(682 \pm 266 \mathrm{~mm}^{3}\right.$ vs. $\left.1,160 \pm 617 \mathrm{~mm}^{3}\right)$ after 6 weeks of intervention (Fig. 4). In fact, the inhibition of the tumor growth was statistically significant from the fourth week onward resulting almost 50\% inhibition at the end of the study compared to the control. In a second study, different doses of BB powder were tested against human A549 lung cancer xenograft, which has a growth rate slower compared with the H1299 cells. Diets supplemented with $2.5 \%$ and $5 \%$ of BB powder were provided (i) pre-inoculation stage - before inoculating with the tumor cells and, (ii) post-inoculation stage/therapeutic mode - after establishment of $\sim 80 \mathrm{~mm}^{3}$ tumors. At the end of 7 weeks, the berry diets showed no effect on the growth inhibition of the tumors when the berry diets were administered in pre-inoculation stage at both doses tested (Fig. 5A). Although 5\% blueberry dose showed slight protective effects, it did not achieve statistical significance. However, the berry diets administered post-inoculation clearly suppressed the tumor growth at both the doses. The 5\% diet showed significant inhibition (64\%) of tumor growth while the $2.5 \%$ diet showed somewhat lesser effect (45\%) inhibition (Fig. 5). 


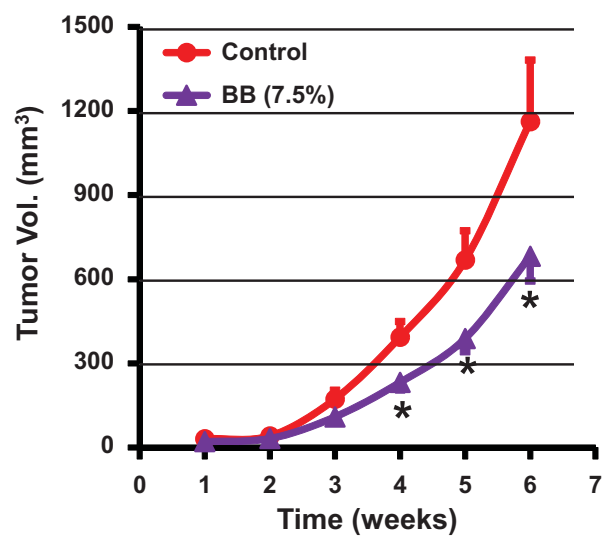

Fig. 4. Antitumor activity of dietary blueberry (BB) powder against lung cancer tumor xenograft in nude mice. Animals were inoculated with human lung cancer H1299 cells $\left(1.5 \times 10^{6}\right.$ cells $)$ and tumor volume was measured weekly until end of the study $(6$ weeks $)$. Mice $(n=9)$ were fed either a control diet (AIN 93M) or diet supplemented with blueberry powder $(7.5 \% \mathrm{w} / \mathrm{w})$. $p$ value $<0.05$ was considered significant (two-tailed Student's $t$ test).
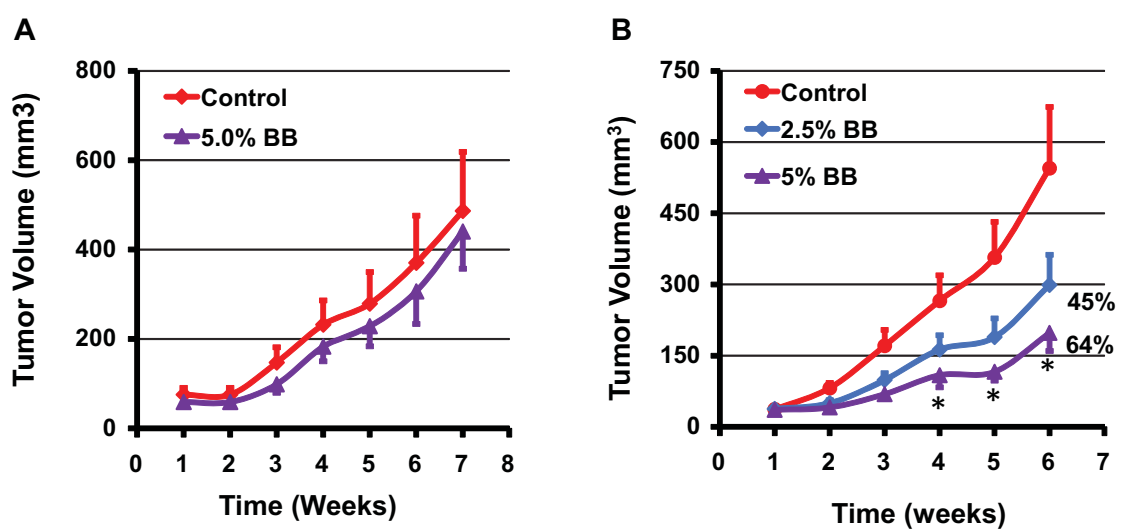

Fig. 5. Effect of diet supplemented with indicated doses of blueberry (BB) powder administered before inoculating the tumor cells (A) and after establishment of the tumor cells (B) on the growth of human A549 lung cancer cell xenograft in nude mice. Mice $(n=8)$ were fed either control diet (AIN 93M) or diet supplemented with blueberry powder ( 2.5 and $5 \% \mathrm{w} / \mathrm{w}$ ) and euthanized after 7 weeks when tumor volume reached $\sim 600 \mathrm{~mm}^{3}$. Data represent mean \pm SE. $p$ value $<0.05$ was considered significant (two-tailed Student's $t$ test).

These findings indicate that diet supplemented with BB powder has varying effects on the growth inhibition of human lung cancer xenograft based on the time of the intervention. The BB diet showed inhibition of the tumor xenograft resulting from both the H1299 and A549 lung cancer cells in 'therapeutic mode'. The 5\% diet had the maximal inhibitory effect with slight or no additional advantage occurring with a higher dose (7.5\%). As two different cell lines were used, $7.5 \%$ dose might have resulted in slightly lower inhibition in view of aggressiveness of the H1299 cell line. It is unclear why the berry diet failed to show any significant effect on the tumor growth when it was administered in pre-inoculation phase. One possible explanation for this lack of protective effect when the berry diet was provided in pre-inoculation phase may be due to low threshold levels of BB bioactives in the plasma at the time of tumor establishment. We have observed in our previous studies that dietary curcumin showed higher levels in plasma up to four days and then settled at much lower concentrations possibly due to 


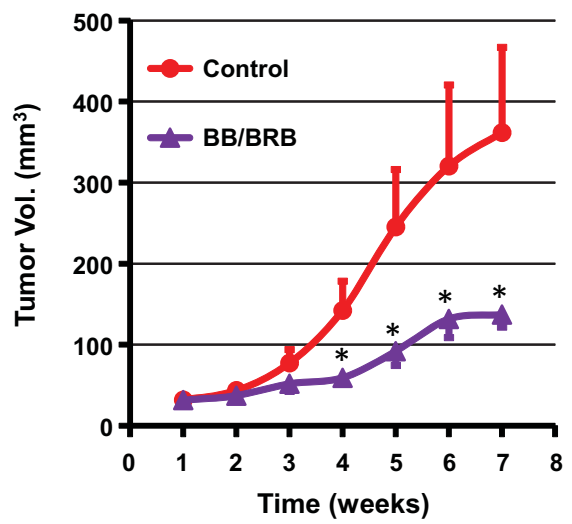

Fig. 6. Antitumor activity of a diet supplemented with both blueberry (BB) and black raspberry (BRB) against lung cancer tumor xenograft in nude mice. Animals were inoculated with human lung cancer A549 cells $\left(2.5 \times 10^{6}\right.$ cells $)$ and tumor volume was measured weekly. Mice $(n=8)$ were fed either a control powder diet (AIN 93M) or diets supplemented with BB powder (5\%) and BRB powder (2.5\%) until the end of the study (7 weeks). $p$ value $<0.05$ was considered significant (two-tailed Student's $t$ test).

systemic adaptive response to xenobiotic metabolism [21]. On the other hand, the pharmacokinetic studies of anthocyanins in rats and humans indicate that peak levels of BRB anthocyanins in blood are reached within one hour after dietary administration and they are cleared from the blood within few hours [22, 23]. More studies on the metabolism of $\mathrm{BB}$ and tissue distribution are warranted to develop better strategies for its utilization for chemoprevention approaches. Unlike strong chemopreventive and therapeutic effects of BB against estrogenmediated breast cancer in ACI rat model reported from our laboratory [20], the protective effect of the berry diet against human lung cancer tumor in the nude mice were modest. On the other hand bioactives from BB/BRB exhibited strong therapeutic activity in this lung cancer model [14], suggesting that the dose of the bioactives from the BB diet may be insufficient.

The outcome of chemopreventive strategies relies largely on the ability of agents to maximally exploit the intrinsic anti-tumor potential without any toxicity. Targeting distinct molecular pathways by a combinatorial approach would be ideal to delay the progression of cancer. Therefore, in our third study, we tested the combination of BB (5\%,w/w) and BRB $(2.5 \%, \mathrm{w} / \mathrm{w})$ against A549 lung tumor xenograft. At the end of 7 weeks, the dietary $\mathrm{BB} / \mathrm{BRB}$ combination resulted in significant reduction of growth of tumor xenograft (63\%) compared to the vehicle control, with a significant growth inhibition starting as early as 4 weeks in the study (Fig. 6). The greater degree of inhibition by this combination possibly resulted from the diverse chemical profiles of BB and BRB. BB contains five distinct anthocyanidins ( $\mathrm{Dp}, \mathrm{Cy}, \mathrm{Pt}, \mathrm{Pe}$, and $\mathrm{Mv}$ ) where as BRB contains almost exclusively $\mathrm{Cy}$ and EA. However, the role of the fibers from BB and BRB in tumor inhibitory activity cannot be completely ruled out as fiber fraction from three different berries have been shown to possess chemoprevention activity against esophageal tumorigenesis [6].

It is becoming increasingly clear that the modulation of multiple pathways would be an effective approach for the prevention and treatment of human cancers, including lung cancer. The use of natural and chemotherapeutic agents acting on different deregulated gene products may represent a potentially better strategy than targeting one specific oncogene target to treat or prevent cancer and disease recurrence [24, 25]. We have reported that the mixture of anthocyanidins from BB affected distinct and overlapping molecular targets in lung cancer leading to enhanced anti-cancer effects [14]. Our current findings indicate BB anthocyanidins in combination with other polyphenolics including EA from BRB resulted in an enhanced anti-tumor effect, corroborating with the presumption of synergism when used together. 
Delphinidin 3-sambubioside has been demonstrated to induce apoptosis in HL-60 through reactive oxygen species (ROS)-mediated mitochondria pathway, suggesting these anthocyanins are good candidates for ROSgenerating reagents [26]. Similarly, cyanidin-3-rutinoside was also shown to generate ROS in leukemia cells to cause cytostatics effects [27]. Therefore, combining these agents with other drugs that act in a ROS-dependent or independent manner is suggested to potentiate the action that contributes to cancer cell killing [28, 29]. Proanthocyanidins on the other hand were shown to enhance the doxorubicin (DOX)-induced anti-tumor effects and reverse drug resistance thus enhancing anti-cancer effects [30,31]. The approach of combining anthocyanidins themselves and in combination with other polyphenolics or chemotherapeutic agents to increase therapeutic response is very exciting. However, in-depth studies are warranted to fully evaluate this potential for the prevention and/or treatment of human lung cancer and other cancers.

In Summary, our results show that the berries and their polyphenolics demonstrate a great degree of therapeutic effects. Results also demonstrate that the anthocyanidins show a higher degree of efficacy than the anthocyanins against lung cancer cells and that the combination of BB and BRB rendered a greater degree of lung tumor growth inhibition than individual entities. It is suggested that the BB diet be tested in a chemically-induced lung cancer model to fully recognize chemopreventive effects of BB constituents and combinations of the berry bioactives be further investigated for mechanistic studies.

\section{Acknowledgments}

This work was supported, in part, by U.S. Highbush Blueberry Council grant, Duggan Endowment, and Helmsley grant. Dr. Gupta holds Agnes Brown Duggan Chair in Oncological Research. Medox, Norway is thankfully acknowledged for providing cyanidin and cyanidin-3-glucosides as kind gift. Mr. Anthony W. Gross, Grad Teaching Assistant, at the University of Louisville Writing Center is acknowledged for editorial corrections.

\section{References}

[1] American Cancer Society, Cancer Facts and Figures. American Cancer society, Atlanta.: 2015.

[2] Saisho S, Yasuda K, Maeda A, Yukawa T, Okita R, Hirami Y, et al. Post-recurrence survival of patients with non-small-cell lung cancer after curative resection with or without induction/adjuvant chemotherapy. Interact Cardiov Th. 2013;16(2):166-72.

[3] Harris GK, Gupta A, Nines RG, Kresty LA, Habib SG, Frankel WL, et al. Effects of lyophilized black raspberries on azoxymethaneinduced colon cancer and 8-hydroxy-2'-deoxyguanosine levels in the Fischer 344 rat. Nutr Cancer. 2001;40(2):125-33.

[4] Stoner GD, Wang LS, Seguin C, Rocha C, Stoner K, Chiu S, et al. Multiple berry types prevent N-nitrosomethylbenzylamine-induced esophageal cancer in rats. Pharm Res. 2010;27(6):1138-45.

[5] Wang LS, Hecht SS, Carmella SG, Yu N, Larue B, Henry C, et al. Anthocyanins in black raspberries prevent esophageal tumors in rats. Cancer prevention research. 2009;2(1):84-93.

[6] Wang LS, Hecht S, Carmella S, Seguin C, Rocha C, Yu N, et al. Berry ellagitannins may not be sufficient for prevention of tumors in the rodent esophagus. Journal of agricultural and food chemistry. 2010;58(7):3992-5.

[7] Aiyer HS, Srinivasan C, Gupta RC. Dietary berries and ellagic acid diminish estrogen-mediated mammary tumorigenesis in ACI rats. Nutr Cancer. 2008;60(2):227-34.

[8] Aiyer HS, Gupta RC. Berries and ellagic acid prevent estrogen-induced mammary tumorigenesis by modulating enzymes of estrogen metabolism. Cancer Prevention Research. 2010;3(6):727-37.

[9] Srivani Ravoori HK, Farrukh Aqil and Ramesh C. Gupta. editor Distinct molecular targets of blueberry and black raspberry in breast cancer prevention. American Association of Cancer Research. 2010; Washington DC.

[10] Aqil F, Vadhanam MV, Jeyabalan J, Cai J, Singh IP, Gupta RC. Detection of anthocyanins/anthocyanidins in animal tissues. J Agric Food Chem. 2014;62(18):3912-8.

[11] Jeyabalan J, Aqil F, Munagala R, Annamalai L, Vadhanam MV, Gupta RC. Chemopreventive and therapeutic activity of dietary blueberry against estrogen-mediated breast cancer. J Agric Food Chem. 2014;62(18):3963-71.

[12] Pandey KB, Rizvi SI. Plant polyphenols as dietary antioxidants in human health and disease. Oxid Med Cell Longev. 2009;2(5):270-8. 
[13] Aqil F, Munagala R, Jeyabalan J, Joshi T, Singh IP, Gupta RC. The Indian Blackberry (Jamun), Antioxidant Capacity, and Cancer Protection. In: Preedy V, editor. Cancer: Oxidative Stress and Dietary Antioxidants. Amsterdam. 2015.

[14] Kausar H, Jeyabalan J, Aqil F, Chabba D, Sidana J, Singh IP, et al. Berry anthocyanidins synergistically suppress growth and invasive potential of human non-small-cell lung cancer cells. Cancer Letters. 2012;325(1):54-62.

[15] Aqil F, Gupta A, Munagala R, Jeyabalan J, Kausar H, Sharma RJ, et al. Antioxidant and antiproliferative activities of anthocyanin/ellagitannin-enriched extracts from Syzygium cumini L. (Jamun, the Indian Blackberry). Nutrition and Cancer. 2012;64(3):428-38.

[16] Jeyabalan J, Aqil F, Munagala R, Annamalai L, Vadhanam MV, Gupta RC. Chemopreventive and Therapeutic Activity of Dietary Blueberry against Estrogen-Mediated Breast Cancer. J Agr Food Chem. 2014;62(18):3963-71.

[17] Aiyer HS, Vadhanam MV, Stoyanova R, Caprio GD, Clapper ML, Gupta RC. Dietary berries and ellagic acid prevent oxidative DNA damage and modulate expression of DNA repair genes. Int J Mol Sci. 2008;9(3):327-41.

[18] Li L, Adams LS, Chen S, Killian C, Ahmed A, Seeram NP. Eugenia jambolana Lam. berry extract inhibits growth and induces apoptosis of human breast cancer but not non-tumorigenic breast cells. J Agric Food Chem. 2009;57(3):826-31.

[19] Munagala R, Kausar H, Munjal C, Gupta RC. Withaferin A induces p53-dependent apoptosis by repression of HPV oncogenes and upregulation of tumor suppressor proteins in human cervical cancer cells. Carcinogenesis. 2011;32(11):1697-705.

[20] Ravoori S, Vadhanam MV, Aqil F, Gupta RC. Inhibition of Estrogen-Mediated Mammary Tumorigenesis by Blueberry and Black Raspberry. J Agr Food Chem. 2012;60(22):5547-55.

[21] Bansal SS, Kausar H, Vadhanam MV, Ravoori S, Gupta RC. Controlled systemic delivery by polymeric implants enhances tissue and plasma curcumin levels compared with oral administration. Eur J Pharm Biopharm. 2012;80(3):571-7.

[22] Tian QG, Giusti MM, Stoner GD, Schwartz SJ. Urinary excretion of black raspberry (Rubus occidentalis) anthocyanins and their metabolites. J Agr Food Chem. 2006;54(4):1467-72.

[23] Stoner GD, Sardo C, Apseloff G, Mullet D, Wargo W, Pound V, et al. Pharmacokinetics of anthocyanins and ellagic acid in healthy volunteers fed freeze-dried black raspberries daily for 7 days. J Clin Pharmacol. 2005;45(10):1153-64.

[24] Ghavami G, Kazemali MR, Sardari S. Informatics of drug synergism in naturally occurring anticancer agents. Recent Patents on Anti-Cancer Drug Discovery. 2011;6(1):26-44.

[25] AlQathama A, Prieto JM. Natural products with therapeutic potential in melanoma metastasis. Natural Product Reports. 2015;32(8):1170-82.

[26] Hou DX, Tong X, Terahara N, Luo D, Fujii M. Delphinidin 3-sambubioside, a Hibiscus anthocyanin, induces apoptosis in human leukemia cells through reactive oxygen species-mediated mitochondrial pathway. Arch Biochem Biophys. 2005;440(1):101-9.

[27] Feng R, Ni HM, Wang SY, Tourkova IL, Shurin MR, Harada H, et al. Cyanidin-3-rutinoside, a natural polyphenol antioxidant, selectively kills leukemic cells by induction of oxidative stress. The Journal of Biological Chemistry. 2007;282(18):13468-76.

[28] Yuan B, Okusumi S, Yoshino Y, Moriyama C, Tanaka S, Hirano T, et al. Delphinidin induces cytotoxicity and potentiates cytocidal effect in combination with arsenite in an acute promyelocytic leukemia NB4 cell line. Oncology Reports. 2015;34(1):431-8.

[29] Trachootham D, Alexandre J, Huang P. Targeting cancer cells by ROS-mediated mechanisms: A radical therapeutic approach? Nature reviews Drug Discovery. 2009;8(7):579-91.

[30] Zhang XY, Bai DC, Wu YJ, Li WG, Liu NF. Proanthocyanidin from grape seeds enhances anti-tumor effect of doxorubicin both in vitro and in vivo. Pharmazie. 2005;60(7):533-8.

[31] Zhang XY, Li WG, Wu YJ, Bai DC, Liu NF. Proanthocyanidin from grape seeds enhances doxorubicin-induced antitumor effect and reverses drug resistance in doxorubicin-resistant K562/DOX cells. Can J Physiol Pharm. 2005;83(3):309-18. 